\title{
Weak-field few-femtosecond VUV photodissociation dynamics of water isotopologues
}

\author{
Arne Baumann, ${ }^{1,2,{ }^{*}}$ Sophia Bazzi, ${ }^{3,4}$ Dimitrios Rompotis, ${ }^{1,5}$ Oliver Schepp, ${ }^{1}$ Armin Azima, ${ }^{1,6,2}$ Marek Wieland,,${ }^{1,6,2}$ \\ Daria Popova-Gorelova, ${ }^{3,2}$ Oriol Vendrell, ${ }^{3,2,7}$ Robin Santra, ${ }^{3,8,2,4}$ and Markus Drescher ${ }^{1,6,2}$ \\ ${ }^{1}$ Institute for Experimental Physics, University of Hamburg, Luruper Chaussee 149, 22761 Hamburg \\ ${ }^{2}$ The Hamburg Centre for Ultrafast Imaging, Luruper Chaussee 149, 22761 Hamburg \\ ${ }^{3}$ Center for Free-Electron Laser Science, DESY, Notkestrasse 85, 22607 Hamburg \\ ${ }^{4}$ Department of Chemistry, University of Hamburg, Grindelallee 117, 20146 Hamburg \\ ${ }^{5}$ Deutsches Elektronensynchrotron, DESY, Notkestrasse 85, 22607 Hamburg \\ ${ }^{6}$ Center for Free-Electron Laser Science, University of Hamburg, Luruper Chaussee 149, 22761 Hamburg \\ ${ }^{7}$ Department of Physics and Astronomy, Aarhus University, Ny Munkegade 120, 8000 Aarhus C, Denmark \\ ${ }^{8}$ Department of Physics, University of Hamburg, Jungiusstrasse 9, 20355 Hamburg
}

(Received 23 March 2017; published 27 July 2017)

\begin{abstract}
We present a joint experimental and theoretical study of the VUV-induced dynamics of $\mathrm{H}_{2} \mathrm{O}$ and its deuterated isotopologues in the first excited state $\left(\tilde{A}^{1} B_{1}\right)$ utilizing a VUV-pump VUV-probe scheme combined with ab initio classical trajectory calculations. 16-fs VUV pulses centered at $161 \mathrm{~nm}$ created by fifth-order harmonic generation are employed for single-shot pump-probe measurements. Combined with a precise determination of the VUV pulses' temporal profile, they provide the necessary temporal resolution to elucidate sub-10-fs dissociation dynamics in the $1+1$ photon ionization time window. Ionization with a single VUV photon complements established strong-field ionization schemes by disclosing the molecular dynamics under perturbative conditions. Kinetic isotope effects derived from the pump-probe experiment are found to be in agreement with our by $a b$ initio classical trajectory calculations, taking into account photoionization cross sections for the ground and first excited state of the water cation.
\end{abstract}

DOI: 10.1103/PhysRevA.96.013428

\section{INTRODUCTION}

The VUV-induced photodissociation reaction of water via the first excited state $\left(\tilde{A}^{1} B_{1}\right)$ has been the subject of an abundance of theoretical and experimental studies, as a prototype for a repulsive, barrierless, adiabatic dissociation reaction. The potential energy surface $[1,2]$ of the first excited state has been calculated with high precision by Staemmler and Palma [1]. This is a widely utilized calculation and has been further improved by different groups [3-5]. Based on these calculations, previous theoretical studies focused on the interpretation of the $\tilde{A}^{1} B_{1}$ absorption spectrum [6-9], as well as on the rotational fine structure of the dissociation products [10]. These calculations are supported by a multitude of experiments, such as high-precision absorption spectroscopy [11], Rydberg-tagging experiments [12,13], photoemission spectroscopy [14], or studies of isotope effects on the dissociation dynamics [6,15-19].

With the availability of laser systems capable of generating femtosecond VUV pulses, experimental studies have been extended to the time domain. Farmanara et al. [20,21] were able to identify an upper bound of 20 fs for the photodissociation time constant in the $\tilde{A}^{1} B_{1}$ state, limited by their temporal resolution in a $155-\mathrm{nm}$ single-color pump-probe

\footnotetext{
*arne.baumann@desy.de

Published by the American Physical Society under the terms of the Creative Commons Attribution 4.0 International license. Further distribution of this work must maintain attribution to the author(s) and the published article's title, journal citation, and DOI.
}

experiment. Furthermore, Trushin et al. [22] performed a VUV-pump multiphoton IR-probe experiment and were able to identify different time windows for this pump-probe scheme, defining the time the molecular wave packet needs to exit the Franck-Condon region ( $1.8 \mathrm{fs}$ ) and leave the $1+5$ photon ionization window (3.5 fs). In addition to these experimental studies Imre and Zhang [8], as well as Henriksen et al. [7], deduced the time a wave packet created by vertical excitation of the $\tilde{A}^{1} B_{1}$ state needs to leave the Franck-Condon region is 5 fs.

To gain further insight into the wave-packet dynamics, the present work utilizes 16-fs, 161-nm pulses [23] to study the photodissociation dynamics of $\mathrm{H}_{2} \mathrm{O}$ and its deuterated isotopologues in the first excited state. By combining the ultrashort 161-nm pulses with a single-shot VUV pump/probe approach [24], we are able to disclose sub-10-fs photodissociation dynamics, while greatly reducing acquisition time by collecting a complete pump-probe data set at the repetition rate of the laser system. In addition to the pump-probe measurement, we experimentally determine the instrument response function by means of an intensity autocorrelation of the VUV pulse, enabling deconvolution of the investigated ultrafast dynamics.

This VUV pump-probe approach complements previous studies relying on multiphoton ionization in the probe step, where a sufficiently high infrared field strength is needed. These strong fields may influence the molecular potentials [25] and affect the intrinsic molecular dynamics, as discussed in our previous study on the photodissociation of $\mathrm{O}_{2}$ [26]. In our weak-field approach these effects, as well as the influence of intermediate states, which might be accessed resonantly in the probe step, are excluded, allowing a simplified theoretical 


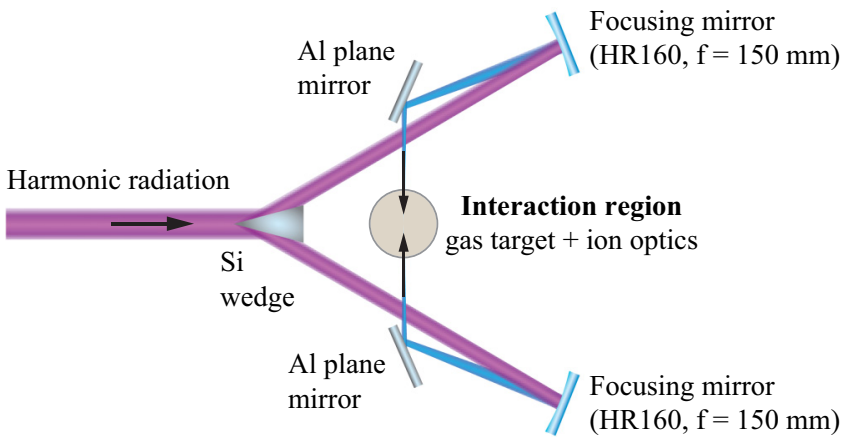

FIG. 1. Sketch of the optical setup used to perform the single-shot pump-probe experiment.

description of the experimental observables for the molecular dynamics. Furthermore, the weak-field case represents a valuable reference for assessing the relevance of strong-field effects at higher intensities, but lower photon energies.

The interpretation of the delay-dependent dynamics of the water isotopologues is supported by a mixed quantum-classical approach to calculate not only the molecular trajectories in the dissociative $\tilde{A}^{1} B_{1}$ state, but also investigate the influence of different final electronic states, accessible by photoionization with a single probe photon, on the observed delay-dependent ion yield. Vertical excitation energies and photoionization cross sections along the reaction coordinate have been calculated, resulting in the delay dependence of the vertical ionization energy from the $\tilde{A}^{1} B_{1}$ state to the electronic ground and first excited states of the ion for an evolving wave packet, which is pivotal in the interpretation of the pump-probe experiment.

\section{METHODS}

\section{A. Experimental methods}

35 -fs pulses with an energy of up to $15 \mathrm{~mJ}$ per pulse delivered by a Ti:Sa laser system relying on chirped-pulse amplification with a central wavelength of $800 \mathrm{~nm}$ and a repetition rate of $25 \mathrm{~Hz}$ were loosely focused into a lengthvariable gas cell to generate high-order harmonic radiation. The generation conditions were optimized for fifth harmonic (161.0-nm, 7.7-eV) pulses with a duration of $16 \mathrm{fs}$ and an energy up to $1.1 \mu \mathrm{J}$ [27].

The single-shot pump-probe technique is described in detail in [24], so here only a short description is provided; a sketch of the optical setup is shown in Fig. 1. The wave front of the harmonic beam was split into two halves by a Si wedge cut at Brewster's angle for $800 \mathrm{~nm}$, suppressing the fundamental pulse and creating a pair of spatially separated VUV pulses. Each VUV pulse followed a separate symmetric beam path, where the fifth harmonic was spectrally purified by focusing multilayer mirrors (HR160 nm, $\mathrm{f}=150 \mathrm{~mm}$, Layertec, $2^{\circ}$ angle of incidence). Both beams were then redirected by Al-coated plane mirrors, bringing the two focused beams in a counterpropagating geometry. A pulsed gas valve provided a gaseous target in the common focal region, where the target was excited and further ionized. The photoionization products were imaged with a time-of-flight ion microscope with a

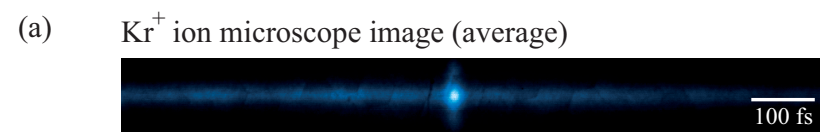

(b)

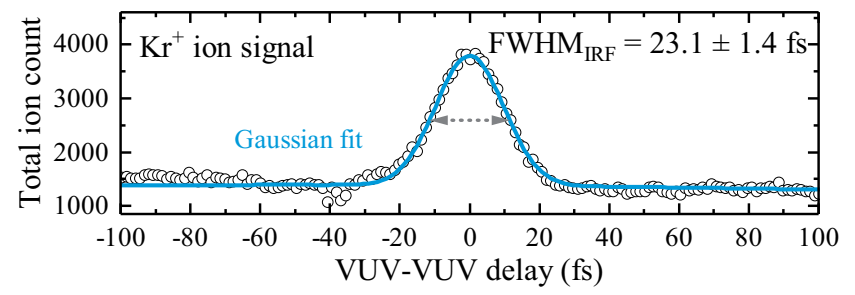

(c) Single-shot intensity autocorrelation

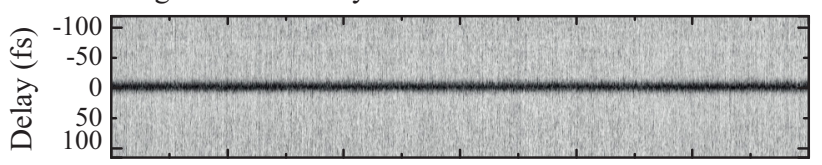

(d)

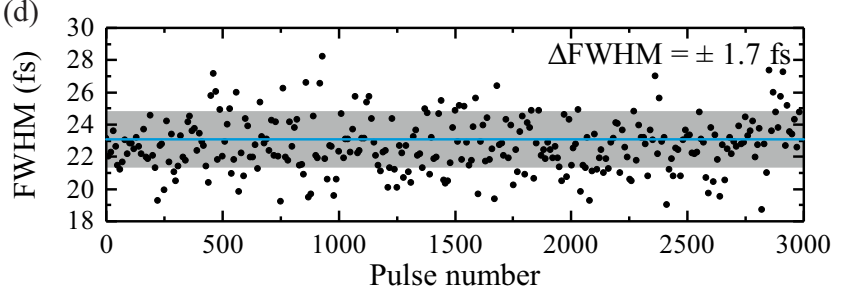

FIG. 2. Ion microscope image and delay-dependent ion signal originating from nonresonant two-photon ionization of Kr. The microscope image (a) shows the averaged spatially resolved $\mathrm{Kr}^{+}$ ion yield for 3000 laser pulses. The averaged delay-dependent $\mathrm{Kr}^{+}$ ion yield (b) corresponds to the VUV pulse intensity autocorrelation, which yields an instrument response function with a FWHM of (23.1 \pm 1.4 ) fs. From the single-shot intensity autocorrelation for each laser pulse (c), the root-mean-square uncertainty of its FWHM (d) can be deduced.

spatial resolution of $<3 \mu \mathrm{m}$ onto a position-sensitive detector, similar to the one described in [28]. Individual ion species were selectively imaged based on their time of flight by applying a high-voltage pulse to the detector, acting as a temporal gate. The temporal delay between the counterpropagating pulses was mapped onto a spatial coordinate in the ion image (see Fig. 2), thus enabling single-shot autocorrelation and pump-probe measurements. Reference measurements with a fringe-resolved interferometric autocorrelation technique [23] were performed using the exact experimental conditions to precisely calibrate the delay-time axis. These measurements resulted in a conversion factor of $(1.3 \pm 0.1) \mathrm{fs} / \mathrm{pixel}$ of the CCD camera used to image the detector.

Furthermore, the pump-probe signal as well as the spatial ion signal of each individual beam path can be observed with the detector in real time, which allows the experimentalist to precisely optimize the spatial overlap between both beam paths and measurement conditions.

Nonresonant two-photon ionization of $\mathrm{Kr}$ was used for single-shot intensity autocorrelation measurements in order to experimentally determine the instrument response function. $\mathrm{H}_{2} \mathrm{O}$ and $\mathrm{D}_{2} \mathrm{O}$ were supplied from the heated vapor of pure liquids, while HDO was supplied from the vapor of a mixture of both. By applying a backing pressure of less than $200 \mathrm{mbar}$ 
(a)

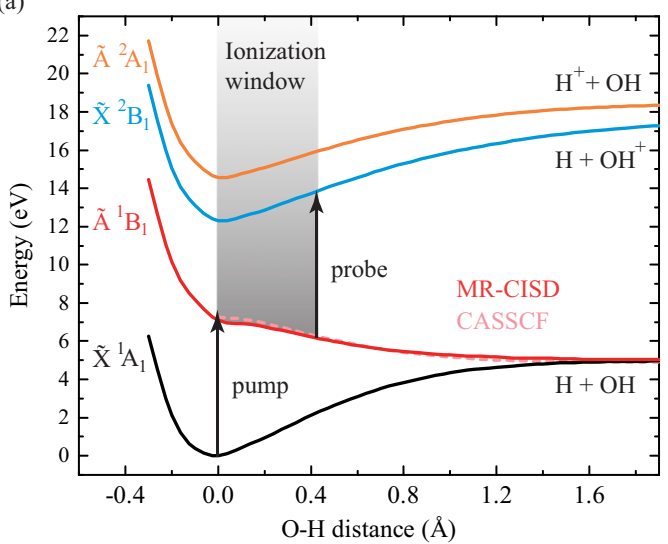

(b)
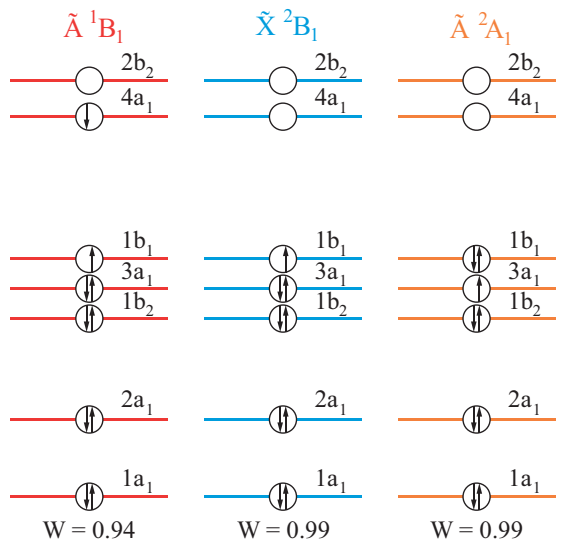

FIG. 3. (a) Cuts through the potential energy surfaces for the ground and first excited electronic states of $\mathrm{H}_{2} \mathrm{O}$ and $\mathrm{H}_{2} \mathrm{O}^{+}$calculated at the MR-CISD $(8,7)$ and MR-CISD (7,7) level, respectively (solid lines), and for the $\tilde{A}^{1} B_{1}$ state of $\mathrm{H}_{2} \mathrm{O}$ calculated with CASSCF(6,4) $($ dashed line). The dissociation coordinate is obtained by changing the length of one of the $\mathrm{O}-\mathrm{H}$ bonds $\left(\mathrm{R}_{\mathrm{OH}}\right)$ and maintaining the other $\mathrm{O}-\mathrm{H}$ bond length and the angle $\mathrm{HOH}$ at the equilibrium values of $0.958 \AA$ and $104.47^{\circ}$, respectively. $\mathrm{R}_{\mathrm{OH}}=0$ corresponds to the equilibrium bond length. (b) The dominant configurations for the $\tilde{A}^{1} B_{1}$ state of a neutral water molecule, and $\tilde{X}{ }^{2} B_{1}$ and $\tilde{A}^{2} A_{1}$ states of the cation and their corresponding CI coefficients at the equilibrium geometry $(\mathrm{W})$ are obtained from the MR-CISD calculation.

the formation of water dimers or clusters could be excluded, verified by ion time-of-flight spectrometry.

\section{B. Computational methods}

The ultrafast dynamics of a water molecule and its deuterated derivatives, $\mathrm{HDO}$ and $\mathrm{D}_{2} \mathrm{O}$, on their first electronically excited state was studied using a mixed quantum-classical approach in which the nuclear motions were treated classically by Newton's equations of motion and the electrons were described quantum mechanically. Since the molecules are sufficiently cold in the experiment, the nuclear coordinates and momenta were sampled from the harmonic Wigner distribution [31] for the ground vibrational level of the $\tilde{X}{ }^{1} A_{1}$ electronic ground state, in which the zero-point vibrational energy is given to each normal mode. It is also assumed that all water molecules are initially in the rotational ground state. In this way initial conditions for 20 classical trajectories on the $\tilde{A}^{1} B_{1}$ potential energy surface were obtained. The trajectories were then divided into two subsets of ten trajectories. Similar temporal evolutions of the two subsets confirmed sufficiently good statistics. For each trajectory 0.1-fs time steps were used for the numerical integration of Newton's equations using the velocity Verlet algorithm [32].

On-the-fly $a b$ initio calculations of the electronic structure, including the gradients, were done with the complete active space self-consistent field method using six active electrons in four active orbitals, $\operatorname{CASSCF}(6,4)$ (complete active space self-consistent field), and the augmented double- $\zeta$ basis set, aug-cc-pVDZ [33,34], utilizing the MOLCAS program package [35]. In order to improve the numerical stability of the CASSCF calculations, the excited-state Hartree-Fock molecular orbitals of the positive ion were used as the initial molecular orbitals. The energies were reevaluated with single point calculations at the multireference single and doubles configuration-interaction level with eight correlated electrons and seven active orbitals, MR-CISD $(8,7)$, and the aug-cc-pVDZ basis set. Both CASSCF and MR-CISD were employed because only CASSCF allows one to compute gradients analytically within MOLCAS, which is optimal for calculating chemical trajectories, whereas MR-CISD provides higher accuracy for ionization energies. Since the analytic gradients are calculated based on the CASSCF potential energy surface, the location of the atoms in space depends on the CASSCF force and therefore it is very crucial to have a correct shape of the CASSCF potential, especially at the FC region for the $\tilde{A}^{1} B_{1}$ state, where the photodissociation reaction of water takes place. The MR-CISD potential is used as a reference to check the validity of the CASSCF potential. The closer the CASSCF potential shape is to the MR-CISD shape, the more reliable the CASSCF potential is [see Fig. 3(a)]. The accuracy of the shape of the MR-CISD potential itself is checked based on the expected vertical ionization values at both the FC region and at the asymptotic region, where one $\mathrm{OH}$ bond is broken (Table I). As it can be seen from Table I, at the FC region, the value of MR-CISD vertical ionization energies from the first electronic excited state of neutral water to the ground and first excited electronic states of water cation obtained from the MR-CISD potential are in a very good agreement with the corresponding experimental values. At the asymptotic region,

TABLE I. Vertical ionization energies of $\mathrm{H}_{2} \mathrm{O}$ at the experimental equilibrium position (top) and at the asymptotic region (bottom) calculated with (a) MR-CISD and (b) CASSCF with the OH bond length kept fixed at the equilibrium $\mathrm{OH}$ bond length of $\mathrm{H}_{2} \mathrm{O}$. Experimental ionization energies for $\mathrm{H}_{2} \mathrm{O}, \mathrm{OH}$ in their respective equilibrium geometries and the hydrogen radical are taken from Refs. [29] and [30]. All values in electronvolts.

\begin{tabular}{lrcr}
\hline \hline Electronic states & (a) Calc. & (b) Calc. & Expt. \\
\hline$\tilde{A}{ }^{1} B_{1} \rightarrow \tilde{X}^{2} B_{1}$ & 5.000 & 4.249 & 5.168 \\
$\tilde{A}^{1} B_{1} \rightarrow \tilde{A}^{2} A_{1}$ & 7.302 & 6.829 & 7.192 \\
$\tilde{A}^{1} B_{1} \rightarrow \tilde{X}^{2} B_{1}\left(\mathrm{H}+\mathrm{OH}^{+}\right)$ & 12.247 & & 13.017 \\
$\tilde{A}^{1} B_{1} \rightarrow \tilde{A}^{2} A_{1}\left(\mathrm{H}^{+}+\mathrm{OH}\right)$ & 13.420 & & 13.598 \\
\hline \hline
\end{tabular}


MR-CISD vertical ionization energy for $\mathrm{H}^{+}+\mathrm{OH}$, which is in principle the ionization energy of a hydrogen radical, is in excellent agreement with the experimental ionization energy [see Table I and Fig. 3(a)]. The ionization energy of $\mathrm{H}+\mathrm{OH}^{+}$ depends on the $\mathrm{OH}$ bond length. The vertical ionization energy of $\mathrm{OH}$ radical, calculated with MR-CISD, deviates from the corresponding experimental value by $0.77 \mathrm{eV}$, which is not a negligible deviation [see Table I and Fig. 3(a)]. This deviation, however, is not due to the inaccuracy of the MR-CISD potential energy surfaces, but it is stemming from the difference between the length of the $\mathrm{OH}$ bond in our calculations with that in the experimental conditions. For the scan underlying Table I, the $\mathrm{OH}$ bond length was kept fixed at the equilibrium $\mathrm{OH}$ bond length of $\mathrm{H}_{2} \mathrm{O}$; therefore its length at asymptotic region differs from its optimal experimental value.

The time windows in which the neutral molecule can be ionized to the electronic ground and first excited states of the cation with a single photon of $7.7 \mathrm{eV}$ energy are obtained by averaging over 20 trajectories. Outside these time windows, the geometry is so far distorted in comparison to the equilibrium geometry that the cation could no longer be created with a single photon of 7.7-eV energy.

Photoionization cross sections $\sigma\left(\tilde{X}^{2} B_{1}\right)$ and $\sigma\left(\tilde{A}^{2} A_{1}\right)$ were calculated for $7.7-\mathrm{eV}$ photon energy as a function of the molecular geometry, applying the orthogonalized plane-wave approximation to the photoelectron wave function [36]. At each molecular geometry the $\operatorname{MR}-\operatorname{CISD}(8,7)$ wave function for the $\tilde{A}^{1} B_{1}$ state and the MR-CISD $(7,7)$ wave functions for the $\tilde{X}^{2} B_{1}$ and $\tilde{A}^{2} A_{1}$ states were used for these calculations.

\section{RESULTS AND DISCUSSION}

The time constant for the VUV photodissociation of $\mathrm{H}_{2} \mathrm{O}$ is expected to be on the order of the duration of the pump-and-probe pulses according to previous studies [22]. To analyze reaction dynamics on this short time scale, a detailed knowledge of the instrument response function, which is given by the second-order intensity autocorrelation (IAC), is necessary. To achieve this, the direct two-photon ionization of $\mathrm{Kr}$ has been measured directly before and after each pump-probe experiment.

The resulting averaged ion image for $\mathrm{Kr}^{+}$is shown in Fig. 2(a) for a data set of 3000 images, exhibiting a pronounced intensity increase where both counterpropagating pulses overlap in space and time. In order to retrieve the intensity autocorrelation of the VUV pulse, the central position of individual ion hits in a single-shot image is extracted to increase the spatial resolution by eliminating the influence of the spot size of individual ion signals on the detector. Considering the delay time-to-space calibration, the delaydependent ion yield is given by the profile of the spatial distribution of the ion image along the propagation axis. The resulting trace, Fig. 2(b), exhibits a peak-to-background ratio near the theoretically expected value of 3:1 for a second-order intensity autocorrelation, which is given by the Gaussian fit (blue). For the shown measurement a FWHM of the intensity autocorrelation of $(23.1 \pm 1.4)$ fs is retrieved, which corresponds to the instrument response function. Although a different temporal profile of the VUV pulse, e.g., a sech ${ }^{2}$ pulse, does result in a shorter retrieved pulse duration, the instrument response function is hardly changed and the possible impact on the pump-probe data sets is negligible.

To reliably distinguish the effect of systematic and statistical uncertainties on the measured pump-probe signal, not only the mean instrument response function is of interest. Utilizing the single-shot capabilities of the colliding-pulse geometry, the effect of shot-to-shot instabilities, such as beam pointing or the temporal stability of the pulses, as well as the long-term stability of the pulse properties become accessible. In Fig. 2(c) the single-shot intensity autocorrelation is shown for each laser pulse. To increase the signal-to-noise ratio a 10-shot average is used to retrieve the temporal jitter of the instrument response function. Figure 2(d) shows the mean FWHM of the instrument response function $(23.1 \pm 1.4) \mathrm{fs}$ as a blue line and its root-mean-square uncertainty as a gray box. Since the root-mean-square uncertainty of $\pm 1.7 \mathrm{fs}$ is comparable to the uncertainty of the average measurement and no temporal drifts of the FWHM are visible, a high stability of the instrument response function over time is ensured.

In contrast to the nonresonant two-photon ionization of $\mathrm{Kr}$, the delay-dependent ion yield for $\mathrm{H}_{2} \mathrm{O}$ and its isotopologues is not only determined by the duration of the VUV pulses, but also by the internal dynamics of the molecule. Figure 3(a) shows a one-dimensional cut through the potential energy surfaces, which are relevant for this pump-probe experiment. The potential energy surfaces have been calculated at MRCISD $(8,7)$ and MR-CISD $(7,7)$ level for $\mathrm{H}_{2} \mathrm{O}$ and $\mathrm{H}_{2} \mathrm{O}^{+}$, respectively. The CASSCF calculation for the $\tilde{A}^{1} B_{1}$ state (light red) is shown in addition, emphasizing the excellent agreement between both calculation methods. In the excitation scheme employed in this pump-probe experiment, the first VUV photon $(7.7 \mathrm{eV})$ excites the molecule from its electronic ground state (black) into the $\tilde{A}^{1} B_{1}$ state (red). Since this state is unbound in the direction of the asymmetric stretch vibration, the molecule starts to dissociate. Not only is the bond distance of the dissociating bond shown in Fig. 3(a) changing during this dissociation reaction, but also the $\mathrm{HOH}$ angle and the second $\mathrm{O}-\mathrm{H}$ bond distance are changing and have been taken into account in the calculation. Due to the increasing energy difference between the excited $\tilde{A}^{1} B_{1}$ state and the electronic ground state of the $\mathrm{H}_{2} \mathrm{O}^{+}$ion (blue), as well as the first excited state (orange), the observation of the dissociation dynamics is confined to a limited time range. Thus, we precisely clock the time when the molecular structure is distorted such that a single VUV-probe photon cannot ionize the molecule anymore [37] [depicted as the gray ionization window in Fig. 3(a)].

The duration of the ionization window can be extracted from the trajectory calculations for each isotopologue. Hence, the vertical excitation energies for the transition from the molecular $\tilde{A}^{1} B_{1}$ state to the electronic ground state and to the first excited state of the ion are calculated for the evolving molecular coordinates after each time step. Figure 4 shows the time evolution of the vertical excitation energies for (a) $\mathrm{H}_{2} \mathrm{O}$, (b) $\mathrm{HDO}$, and (c) $\mathrm{D}_{2} \mathrm{O}$, as well as the photon energy of the probe pulse as a reference. As soon as the excitation energy for each transition exceeds the probe photon energy, the ionization window for the respective excitation scheme closes. The shortest window for ionization to the ground cationic state corresponds to $\mathrm{H}_{2} \mathrm{O}$ and is $6.4 \mathrm{fs}$, increasing to $8.2 \mathrm{fs}$ for 

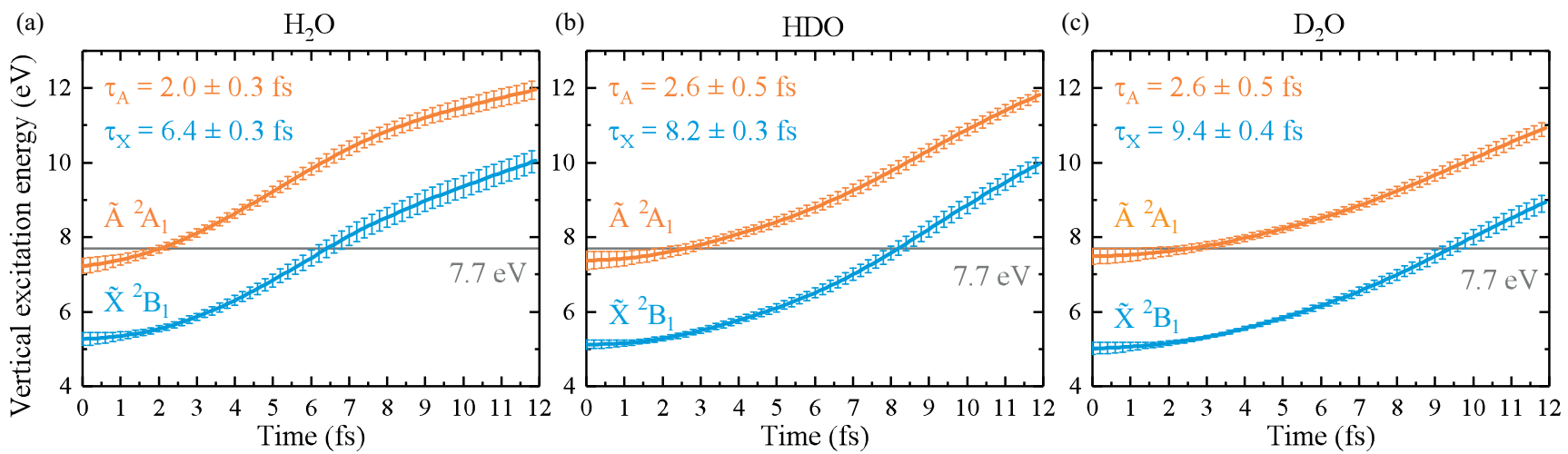

FIG. 4. Calculated time evolution of the vertical excitation energy from the $\tilde{A}^{1} B_{1}$ state to the $\tilde{X}^{2} B_{1}$ state (blue) and from the $\tilde{A}{ }^{1} B_{1}$ state to the $\tilde{A}^{2} A_{1}$ state (orange) during the dissociation for (a) $\mathrm{H}_{2} \mathrm{O}$, (b) $\mathrm{HDO}$, and (c) $\mathrm{D}_{2} \mathrm{O}$ and the corresponding time windows in which ionization from $\tilde{A}^{1} B_{1}$ to $\tilde{X}^{2} B_{1}$ and $\tilde{A}^{2} A_{1}$ with a single photon of $7.7 \mathrm{eV}$ energy (gray line) is possible. The error bars represent the root-mean-square width of the energy distribution for a given time.

$\mathrm{HDO}$, due to the higher mass of the fragment. $\mathrm{D}_{2} \mathrm{O}$ exhibits the longest time window of $9.4 \mathrm{fs}$.

The corresponding ionization window for a probe transition to the first electronically excited state of $\mathrm{H}_{2} \mathrm{O}^{+}$and its deuterated derivatives is much shorter than the time window for ionization to their ground electronic state. Depending on the ratio between their respective photoionization cross sections, both probe transitions may contribute to the experimentally observed ionization window. The longest ionization window is observed if the photoionization cross section $\sigma$ for the $\tilde{X}^{2} B_{1}$ channel is much larger than $\sigma\left(\tilde{A}^{2} A_{1}\right)$. On the other hand, the apparent ionization window gets shorter as the ratio $\sigma\left(\tilde{A}^{2} A_{1}\right) / \sigma\left(\tilde{X}^{2} B_{1}\right)$ increases. It should be noted that, even at distances where ionization is still possible, $\sigma$ is not necessarily constant.

According to our calculations, $\sigma\left(\tilde{A}^{2} A_{1}\right)$ is negligible in comparison to $\sigma\left(\tilde{X}^{2} B_{1}\right)$ at all molecular geometries where ionization to both states is possible. This can even be demonstrated at the independent-particle level, which implies that a single photon can remove an electron from a configuration only without influencing the rest of the configuration. Figure 3(b) shows the dominant configurations for the $\tilde{A}^{1} B_{1}$ state of $\mathrm{H}_{2} \mathrm{O}$ and the $\tilde{X}^{2} B_{1}$ and $\tilde{A}^{2} A_{1}$ states of $\mathrm{H}_{2} \mathrm{O}^{+}$and their corresponding MR-CISD coefficients at the equilibrium geometry. It can be seen from Fig. 3(b) that a transition from the dominant configuration of $\tilde{A}^{1} B_{1}$ to the dominant configuration of $\tilde{X}^{2} B_{1}$ is possible at the independent-particle level, because only one electron is removed in the single-photon probe step. In contrast, the transition to the dominant configuration of $\tilde{A}^{2} A_{1}$ is forbidden, since an additional electron is excited to another orbital, requiring a two-photon absorption. Other configurations of $\tilde{A}^{1} B_{1}$ provide a negligible contribution to the photoionization probability to the state $\tilde{A}^{2} A_{1}$. The configurations shown in Fig. 3(b) are dominant for the corresponding states at all geometries where photoionization is possible. Thus, since $\sigma\left(\tilde{A}^{2} A_{1}\right)$ is negligible in comparison to $\sigma\left(\tilde{X}^{2} B_{1}\right)$, the photoionization time windows are determined exclusively by the vertical excitation energy from the $\tilde{A}^{1} B_{1}$ to the $\tilde{X}^{2} B_{1}$ potential energy surface in the employed single-photon probe scheme. For a given pump photon energy the time evolution shown in Fig. 3(a) is universal and the $\widetilde{A}^{2} A_{1}$ state of the ion will be relevant for a multiphoton probe scheme.
The experimentally obtained pump-probe signal for $\mathrm{H}_{2} \mathrm{O}$ and its deuterated derivatives is determined by the convolution of the instrument response function and the internal dynamics of the respective molecule. To deconvolve these dynamics from the experimental data sets, where the instrument response function is on the same time scale as the expected ionization window, an appropriate fit function needs to be applied. As has been shown in our trajectory simulations, the first trajectories start leaving the ionization window after more than $5 \mathrm{fs}$ and up until this time the population in the ionization window stays constant. This behavior can be modeled by a rectangular function, where the duration of the ionization window is given by its half-width. The convolution of this function and the instrument response function, fitted with a Gaussian, is given by

$$
I(\Delta t)=a\left[\operatorname{erf}\left(\frac{\tau_{1 / 2}-\Delta t}{\sqrt{2} \sigma_{p}}\right)+\operatorname{erf}\left(\frac{\tau_{1 / 2}+\Delta t}{\sqrt{2} \sigma_{p}}\right)\right] .
$$

Here $\Delta t$ is the delay between pump and probe pulse and $\sigma_{p}$ is the standard deviation of the instrument response function $\left[\sigma_{p}=\mathrm{FWHM} /(2 \sqrt{2 \ln 2})\right]$, while $\tau_{1 / 2}$, the half-width of the rectangular function, and the scaling factor $a$ are the free fit parameters.

For $\mathrm{H}_{2} \mathrm{O}$, with a corresponding FWHM of $(22.3 \pm 1.3)$ fs for the instrument response function, the fit yields $\tau_{1 / 2}=$ $(6.7 \pm 1.8)$ fs [Fig. 5(b)]. For HDO a slightly narrower instrument FWHM of $(21.4 \pm 1.3)$ fs for the instrument response function results in $\tau_{1 / 2}=(7.5 \pm 1.7)$ fs. The internal dynamics in $\mathrm{D}_{2} \mathrm{O}$ are proceeding slower, which leads to a stronger deviation from the intensity autocorrelation with a FWHM of $(20.3 \pm 1.2)$ fs. $\tau_{1 / 2}$ is $(10.4 \pm 1.5)$ fs in this case, which is consistent with a primary kinetic isotope effect of $\sqrt{2}$ from doubling the fragment mass. When an exponential decay function is used to deconvolve the duration of the ionization window, its duration is underestimated, because the delayed decrease of population in the ionization window is not considered. Slight differences of the instrument response function in the individual pump-probe experiments are attributed to the daily optimization of the laser system. By obtaining consecutive measurements it was ensured that the pulse duration was stable over the time the pump-probe measurement was performed. 
(a)

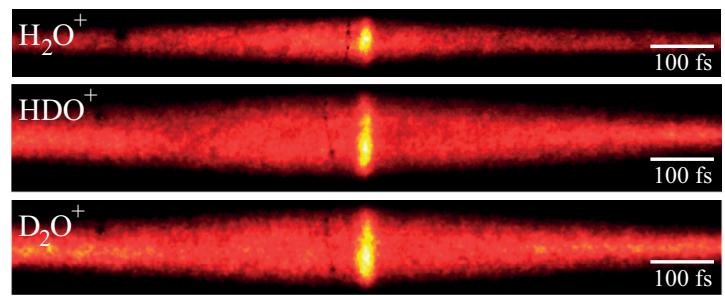

(b) $\begin{array}{rrrrrrrrrrr}-100 & -80 & -60 & -40 & -20 & 0 & 20 & 40 & 60 & 80 & 100\end{array}$
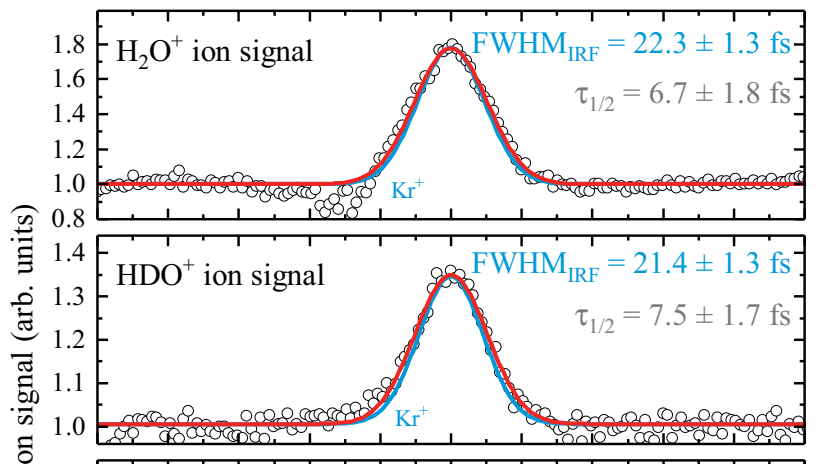

ธิ

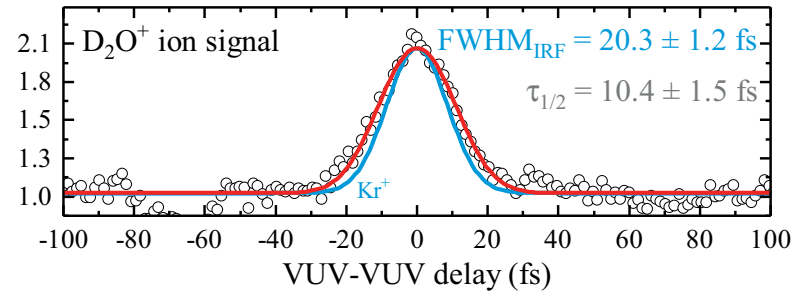

FIG. 5. Ion microscope images and delay-dependent ion signals for $\mathrm{H}_{2} \mathrm{O}, \mathrm{HDO}$, and $\mathrm{D}_{2} \mathrm{O}$. The microscope image (a) shows the averaged spatially resolved ion yield of the different isotopologues for 3000 laser pulses. The delay-dependent ion signal (b) of each isotopologue is shown in comparison to the instrument response function (blue) recorded in the corresponding measurement series and fitted with a Gaussian function (red). Depending on the degree of deuterization, an increased FWHM is observed in respect to the instrument response function, indicating a kinetic isotope effect on the excited-state dynamics of the molecule.

For all isotopologues an excellent agreement between the duration of the ionization window deconvolved from the delay-dependent ion yield and the duration of the ground-state ionization window derived from the mixed quantum-classical calculation was achieved, showing that ionization to the ionic ground state is the only significant probe transition contributing to the delay-dependent parent ion yield.

These results can be compared to existing multiphoton probe studies to discuss the effect of the strong-field probe on the intrinsic dynamics. Trushin et al. [22] extract a time constant of 3.5 fs for $\mathrm{H}_{2} \mathrm{O}$ from a exponential decay model for the dynamics in the $1+5$ photon ionization window, which is comparable to the window in the present study. The derivation of time constants from experimental pump-probe data sets is strongly influenced by the respective model used (exponential decay model compared to a rectangular window in the present study). If the exponential decay is used to evaluate the present delay-dependent data, a time constant of $(4.0 \pm 2.2)$ fs is extracted for $\mathrm{H}_{2} \mathrm{O}$, which agrees with the multiphoton probe result to within $15 \%$. Due to the precision of the measurement, a systematic effect of strong-field ionization in respect to our weak-field approach cannot be excluded.

Another key result of this joint experimental and theoretical study is that the interpretation of pump-probe data even for a "simple" dissociation reaction in the sub-10-fs regime is not trivial. Even when the instrument response function can be determined precisely, the choice of an appropriate model function for the delay dependence of the observable is necessary-in this case the time the molecule needs to exit the ionization window. Assuming an exponential decay rate for the observable, as it is valid for most reactions studied by femtochemistry on the few-hundred-femtosecond or picosecond time scale, leads in this case to an underestimation of the duration of the ionization window, while the deconvolved duration of a rectangular window coincides well with our mixed quantum-classical trajectory calculation.

\section{CONCLUSION}

The photodissociation dynamics of $\mathrm{H}_{2} \mathrm{O}$ and its isotopologues in the $\tilde{A}^{1} B_{1}$ state have been studied in a combined single-shot autocorrelation and pump-probe experiment relying on a VUV-pump-VUV-probe scheme at 7.7-eV photon energy. This constitutes a measurement with sufficient time resolution and a single-color approach which does not perturb the system under study, in contrast to multiphoton IR-probe schemes. Furthermore, our single-shot approach allows us to drastically reduce the acquisition time compared to delayscanning techniques, reducing the influence of long-term instabilities. By determining the instrument response function under the same conditions as the pump-probe experiment for single shots, it is possible to ensure a high stability of the pulse duration of pump and probe pulses and temporal drifts can be excluded.

The experiments are accompanied by ab initio calculations, which allowed us to identify the relevant probe transition scheme. From two possible transitions, the excitation to the ionic ground state is the relevant probe transition. The expected duration of the $1+1$ photon ionization window was predicted by calculating the time-dependent vertical excitation energies for an evolving wave packet in the $\tilde{A}^{1} B_{1}$ state. The experimentally deduced time of 6.7 fs the $\mathrm{H}_{2} \mathrm{O}$ molecule takes to leave the single-photon ionization window while dissociating, as well the influence of hydrogen substitution ( 7.5 fs for $\mathrm{HDO}$ and 9.4 fs for $\mathrm{D}_{2} \mathrm{O}$ ), are consistent with the trajectory calculations using the ground-state ionization window.

\section{ACKNOWLEDGMENTS}

This work has been supported by the Deutsche Forschungsgemeinschaft within the GRK 1355 (Physics with new coherent light sources), the SFB 925 (Light induced dynamics and control of correlated quantum systems), and the excellence cluster "The Hamburg Centre for Ultrafast Imaging - Structure, Dynamics, and Control of Matter at the Atomic Scale," Subprojects A2 and A5. We thank the BMBF for support through the Project 05K10GUA. 
[1] V. Staemmler and A. Palma, Chem. Phys. 93, 63 (1985).

[2] V. Engel, R. Schinke, and V. Staemmler, J. Chem. Phys. 88, 129 (1988).

[3] A. J. Dobbyn and P. J. Knowles, Mol. Phys. 91, 1107 (1997).

[4] R. van Harrevelt and M. C. van Hemert, J. Chem. Phys. 112, 5777 (2000).

[5] R. van Harrevelt and M. C. van Hemert, J. Chem. Phys. 114, 9453 (2001).

[6] V. Engel and R. Schinke, J. Chem. Phys. 88, 6831 (1988).

[7] N. E. Henriksen, J. Zhang, and D. G. Imre, J. Chem. Phys. 89, 5607 (1988).

[8] D. G. Imre and J. Zhang, Chem. Phys. 139, 89 (1989).

[9] F. Xu, L. Wang, C. C. Martens, and Y. Zheng, J. Chem. Phys. 138, 024103 (2013).

[10] L. Zhou, D. Xie, Z. Sun, and H. Guo, J. Chem. Phys. 140, 024310 (2014).

[11] R. Mota, R. Parafita, A. Giuliani, M. J. Hubin-Franskin, J. M. C. Lourenço, G. Garcia, S. V. Hoffmann, N. J. Mason, P. A. Ribeiro, M. Raposo, and P. Limão-Vieira, Chem. Phys. Lett. 416, 152 (2005).

[12] X. F. Yang, D. W. Hwang, J. J. Lin, and X. Ying, J. Chem. Phys. 113, 10597 (2000).

[13] I.-C. Lu, F. Wang, K. Yuan, Y. Cheng, and X. Yang, J. Chem. Phys. 128, 066101 (2008).

[14] J. Z. Zhang, E. H. Abramson, and D. G. Imre, J. Chem. Phys. 95, 6536 (1991).

[15] N. Shafer, S. Satyapal, and R. Bersohn, J. Chem. Phys. 90, 6807 (1989).

[16] R. L. Vander Wal and F. F. Crim, J. Phys. Chem. 93, 5331 (1989).

[17] R. L. Vander Wal, J. L. Scott, F. F. Crim, K. Weide, and R. Schinke, J. Chem. Phys. 94, 3548 (1991).

[18] J. Zhang, D. G. Imre, and J. H. Frederick, J. Phys. Chem. 93, 1840 (1989).

[19] N. Engholm Henriksen and B. Amstrup, Chem. Phys. Lett. 213, 65 (1993).
[20] P. Farmanara, O. Steinkellner, M. Wick, M. Wittmann, G. Korn, V. Stert, and W. Radloff, J. Chem. Phys. 111, 6264 (1999).

[21] M. Wittmann, M. Wick, O. Steinkellner, P. Farmanara, V. Stert, W. Radloff, G. Korn, and I. Hertel, Opt. Commun. 173, 323 (2000).

[22] S. A. Trushin, W. E. Schmid, and W. Fuß, Chem. Phys. Lett. 468, 9 (2009).

[23] T. Gebert, D. Rompotis, M. Wieland, F. Karimi, A. Azima, and M. Drescher, New J. Phys. 16, 073047 (2014).

[24] D. Rompotis, A. Baumann, O. Schepp, T. Maltezopoulos, M. Wieland, and M. Drescher, Optica 4, 871 (2017).

[25] J. H. Posthumus, Rep. Prog. Phys. 67, 623 (2004).

[26] O. Schepp, A. Baumann, D. Rompotis, T. Gebert, A. Azima, M. Wieland, and M. Drescher, Phys. Rev. A 94, 033411 (2016).

[27] D. Rompotis, T. Gebert, M. Wieland, F. Karimi, and M. Drescher, Opt. Lett. 40, 1675 (2015).

[28] M. Schultze, B. Bergues, H. Schröder, F. Krausz, and K. L. Kompa, New J. Phys. 13, 033001 (2011).

[29] L. Karlsson, L. Mattsson, R. Jadrny, R. G. Albridge, S. Pinchas, T. Bergmark, and K. Siegbahn, J. Chem. Phys. 62, 4745 (1975).

[30] W. Haynes, CRC Handbook of Chemistry and Physics, 91st ed. (Taylor \& Francis Group, London, 2010).

[31] E. Wigner, Phys. Rev. 40, 749 (1932).

[32] W. C. Swope, H. C. Andersen, P. H. Berens, and K. R. Wilson, J. Chem. Phys. 76, 637 (1982).

[33] R. A. Kendall, T. H. Dunning, Jr., and R. J. Harrison, J. Chem. Phys. 96, 6796 (1992).

[34] D. E. Woon and T. H. Dunning, Jr., J. Chem. Phys. 98, 1358 (1993).

[35] F. Aquilante, L. De Vico, N. Ferré, G. Ghigo, P.-Å. Malmqvist, P. Neogrády, T. B. Pedersen, M. Pitoňák, M. Reiher, B. O. Roos, L. Serrano-Andrés, M. Urban, V. Veryazov, and R. Lindh, J. Comput. Chem. 31, 224 (2010).

[36] J. W. Rabalais, T. P. Debies, J. L. Berkosky, J.-T. J. Huang, and F. O. Ellison, J. Chem. Phys. 61, 529 (1974).

[37] A. Peralta Conde, J. Kruse, O. Faucher, P. Tzallas, E. P. Benis, and D. Charalambidis, Phys. Rev. A 79, 061405 (2009). 